\title{
1D SIMULATIONS OF THERMALLY BUFFERED PRISMATIC BATTERIES THROUGH THE APPLICATION OF PCMS
}

\author{
Ilya T'Jollyn ${ }^{1,2^{*}}$, Jasper Nonneman ${ }^{1,2}$, Wim Beyne ${ }^{1,2}$, Michel De Paepe ${ }^{1,2}$ \\ ${ }^{1}$ Ghent University, Sint-Pietersnieuwstraat 41, Ghent B-9000, Belgium \\ ${ }^{2}$ Flanders Make, Oude Diestersebaan 133, Lommel B-3920, Belgium
}

\begin{abstract}
Thermal management of Li-ion batteries is critical for its performance and lifetime. Furthermore, when batteries are submitted to excessive temperatures by a bad thermal management system, thermal runaway can occur which can destroy the afflicted cell and the adjacent cells in a battery pack. Batteries are subject to cyclic behavior, charging and discharging, which is accompanied by a non-steady-state heat dissipation. Through thermal buffering, heat can be stored temporarily, which allows the heat transfer to the environment to be more evenly and thus reducing the maximal cooling load. Phase change materials or PCMs for thermal buffering are studied in this paper. By melting and solidifying, these substances take up and release a large amount of heat in a small volume and mass. To be able to design a thermal buffering system with PCMs, a one-dimensional transient model is developed to identify which influence design parameters have on the battery temperature. Simulations are performed for pure PCMs and for PCMs enhanced with three types of thermally conducting structures: metal foam, expanded graphite and carbon fibers. The results show that the effectiveness of thermal buffering is highly dependent on the cycle duration. For long cycles in the order of one day or more, thermal buffering can reduce peak temperature by around $4^{\circ} \mathrm{C}$. For medium duration cycles in the order of several hours, peak temperatures can be reduced by around $13^{\circ} \mathrm{C}$. For shorter cycles, heat buffering in the simulated cases was only slightly beneficial for the battery temperature. Furthermore, the simulations show that thermal buffering for battery packs requires a relatively small amount of PCM which results in short heat paths through the PCM. Enhancing the thermal conductivity by using thermally conductive structures slightly improves the thermal buffering performance, but might not be advisable due to the added complexity and cost.
\end{abstract}

KEY WORDS: Electronic equipment cooling, Thermal management, Thermal buffering, Electrical batteries

\section{INTRODUCTION}

With the rising demand for electrified transportation and electrical energy storage, electrical batteries have gone through many developments in the latest years. This has increased the power and energy density of batteries, which is especially advantageous for electric vehicles, where space and weight requirements are very stringent. With higher power densities, higher heat dissipation rates in batteries occur, which increases the temperature of batteries in battery packs. These higher temperatures can have several drawbacks such as reduced electrical efficiency, increased battery ageing and high risk of failure due to thermal runaway [1]. The optimal battery temperature is around $20^{\circ} \mathrm{C}-30^{\circ} \mathrm{C}$, thermal runaway can start from $80^{\circ} \mathrm{C}$. Above $40^{\circ} \mathrm{C}$, a temperature increase of $10^{\circ} \mathrm{C}$ of the battery relates to a decrease in lifetime of battery in the order of $50 \%$. Adding active cooling to battery packs or increasing the cooling power can combat these shortcomings. However, this comes at a cost of increased volume and weight and requires extra energy use.

Since the loading of batteries is inherently transient due to charge-discharge cycles, heat dissipation rates and battery temperatures are also transient. Reducing peak loads and corresponding peak temperatures is beneficial for batteries. A thermal buffering system can achieve this peak temperature reduction without increasing the 
cooling demand, through buffering the dissipated heat and transferring it to the environment with a more constant cooling load. Phase change materials (PCMs) have been proposed as thermal buffers because of their high volumetric energy density [2]. This paper presents the results of one-dimensional simulations of thermally buffered battery cells with PCM and the influence of several boundary conditions and design parameters on the performance of the thermal buffering system.

\section{BATTERY THERMAL BUFFERING UTILIZING PCM}

Al Hallaj and Selman proposed a thermal management system using phase change materials [3]. An array of cylindrical batteries is simulated, where the voids in between the batteries are filled with PCM. Their results show a significantly lower temperature rise of the battery when compared to a battery without PCM under the same conditions. Furthermore, they state that the PCM thermal management system can also be beneficial in cold climates, where the battery temperature is kept for a longer time at a higher level, increasing its overall efficiency.

Although the PCMs increase the thermal mass of the system, they also introduce an additional thermal resistance for the cooling of the batteries. Since PCMs have rather low thermal conductivity (order of magnitude $0,2 \mathrm{~W} / \mathrm{mK}$ ), research has been focused on the combination of structures with PCM to improve the overall thermal conductivity. Khateeb et al. performed experiments on battery packs with PCM and with PCM immersed in an open metal foam (MF) structure [4]. They perceived that utilizing PCMs resulted in a decrease of $45 \%$ in temperature rise of the battery. Using metal foam in the PCM decreased the temperature rise by another $12,5 \%$. Kizilel et al. simulated the performance of a battery pack in contact with a PCM-expanded graphite (EG) matrix [5]. The results showed that heat was absorbed and quickly spread over the matrix, thereby achieving a uniform temperature in the battery pack. Further simulations showed that if one of the cells reaches thermal runaway conditions, propagation of the effect to other cells is negated by the PCM absorbing large quantities of heat with a minor temperature rise. Another possibility to enhance thermal conductivity proposed in literature is to use carbon fibers (CF) in the PCM [6].

Although research has been performed on thermally buffered batteries using PCM, the optimal design of such a thermal management system is not yet studied. This paper focuses on thermal modelling of the battery pack under different conditions. The applicability of the thermal buffering strategy for different applications is investigated through the use of different load cycles for the batteries. A daily cycle is typically expected when utilizing the battery for a household application, while shorter cycles are expected for applications such as electric vehicles. Through the modeling, the optimal amount of PCM and PCM melting temperature is determined for different load cycles. Furthermore, the addition of thermally conductive structures to the PCM is simulated to assess the applicability of the different structures in thermally buffered battery packs.

\section{BATTERY AND PCM THERMAL MODELLING}

3.1 Battery modelling The battery chosen in the modelling is the polymer lithium-ion battery TENERGY, model 30123 (Figure 1). It is a prismatic pouch-type cell. The main battery characteristics are given in Table 1. The thermal characteristics of the battery cell were determined experimentally by Nieto et al. [7].

Table 1 TENERGY 30123 battery characteristics

\begin{tabular}{lrl}
\hline Property & Value & Unit \\
\hline Width & 0,0595 & $\mathrm{~m}$ \\
Height & 0,1570 & $\mathrm{~m}$ \\
Thickness & 0,0098 & $\mathrm{~m}$ \\
Weight & 0,210 & $\mathrm{~kg}$ \\
Nominal electric capacity & 10 & $\mathrm{Ah}$ \\
Max continuous discharge current & 50 & $\mathrm{~A}$ \\
Max continuous charge current & 10 & $\mathrm{~A}$ \\
Heat capacity [7] & 1015 & $\mathrm{~J} / \mathrm{kgK}$ \\
\hline
\end{tabular}

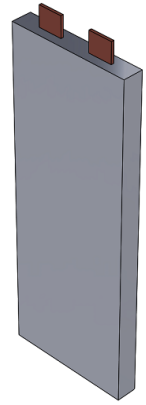

Fig. 1 TENERGY 301323 battery 
A battery pack consists of several battery cells. In the simulations, it is assumed that all battery cells are identical and that there is no thermal interaction between two different cells. Therefor the simulation can be simplified to the simulation of a single battery cell. To model the thermal behavior of a battery, the heat generation behavior of the battery $\mathrm{q}_{\mathrm{b}}$ has to be determined. Two heat generating effects are evaluated: irreversible ( $\mathrm{q}_{\mathrm{irr}}$ ) and reversible ( $\left.\mathrm{q}_{\mathrm{rev}}\right)$ heat. The irreversible heat is due to the Joule losses from the current flowing through the battery, which always result in heat generated by the cell. The reversible heat is related to the entropy change in the battery caused by chemical reactions taking place in the battery during charging and discharging. These chemical reactions can generate heat but also take up heat from the battery. The complete heat generation behavior is described by equations 1 to 3 .

$$
\begin{gathered}
q_{b}=q_{i r r}+q_{r e v} \\
q_{i r r}=R I^{2} \\
q_{r e v}=-I T_{b} \frac{\partial E}{\partial T_{b}}
\end{gathered}
$$

The irreversible heat is squared with the current I and linear with the internal electrical resistance $\mathrm{R}$. This resistance is based on the measurements by Nieto et al., and is dependent on the battery temperature [7]. The reversible heat varies linearly with the current, the absolute temperature of the battery $T_{b}$ and the entropic coefficient, which is the derivative of the open-circuit voltage $\mathrm{E}$ to the battery temperature. The entropic coefficient is also gathered from Nieto et al. and is dependent on the state of charge (SoC) of the battery [7].

Both the reversible and irreversible heat effect are dependent on the current or load profile of the battery. Three different load cycles are analyzed, based on the duration of the load cycle: a long cycle (daily), a medium duration cycle ( 72 minutes) and a short cycle (12 minutes). In the long cycle, the current is far below the maximum rated current of the battery, while it is completely charged and discharged in the course of a day. This is a representative load cycle for a household. In the medium cycle, the current is equal to the maximum rated current and the battery is fully charged and discharged during the cycle, which is representative for several traction applications. In the short cycle, the maximum rated current is drawn from the battery, but only part of the total energy capacity of the battery is used, which is representative for applications of electric grid power management. The current and SoC for the three load cycles are shown in Figures 2, 3 and 4. The simulations use a periodic boundary conditions for the initial state of the system, indicating that the beginning and end state of one cycle should be identical. This corresponds to a simulation of a battery pack that is continuously running according to the load cycle.

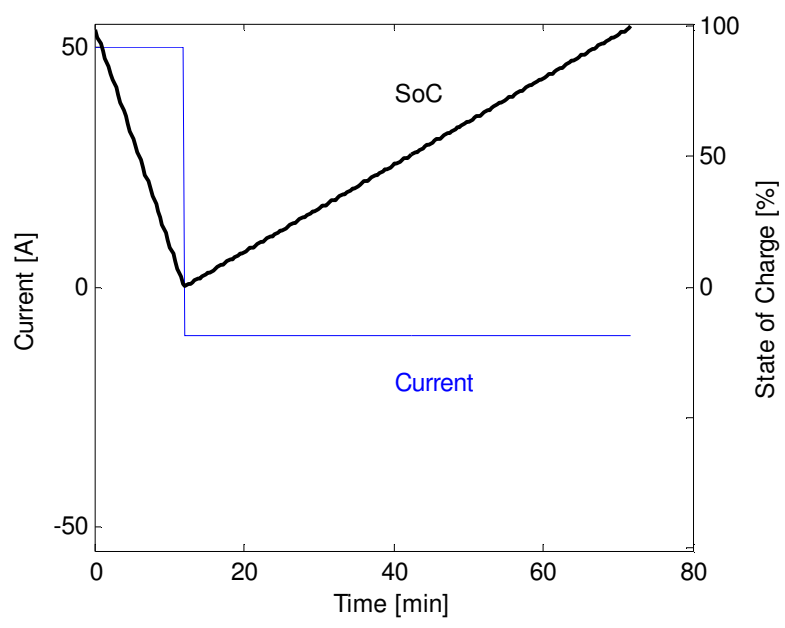

Fig. 2 Current and SoC for the medium duration cycle

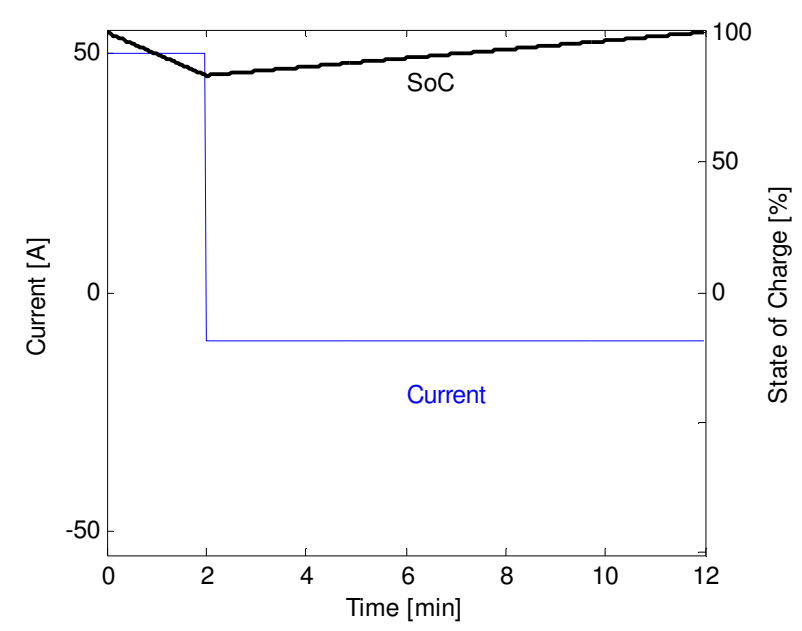

Fig. 3 Current and SoC for the short load cycle 


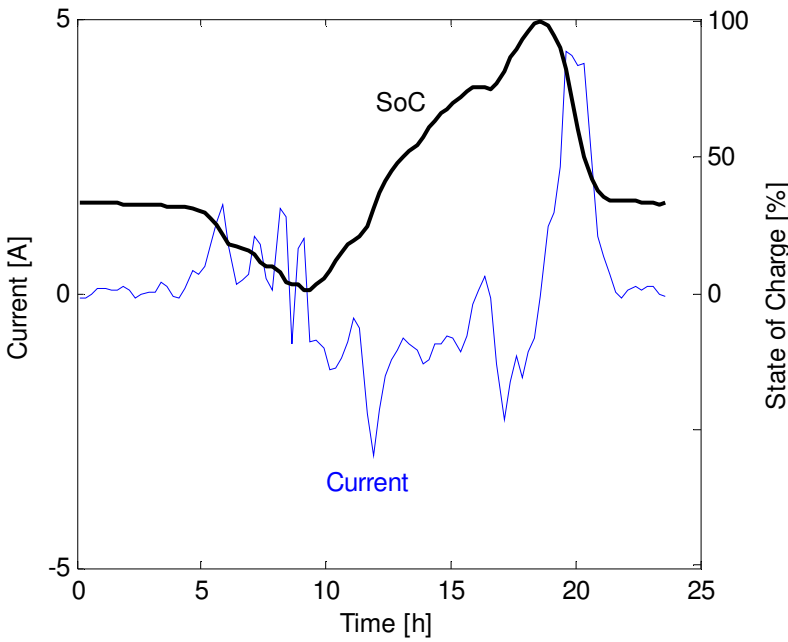

Fig. 4 Current and SoC for the daily cycle

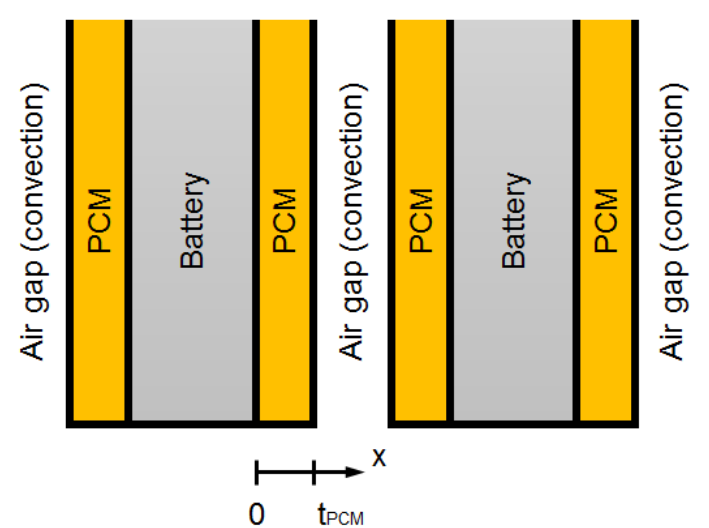

Fig. 5 Cross section of two batteries with PCM thermal buffering (not to scale)

Heat is assumed to be uniformly generated in the battery volume. Since the battery is thickness is small compared to the other dimensions, it is assumed that all heat is transferred through the side surfaces and that the thermal problem can be assessed as a 1D-problem. This shape of battery is preferred for thermal management when compared with other battery geometries, since due to the limited thickness, the thermal resistance of the battery itself can be neglected compared to the thermal resistances of the PCM and the convective resistance. This is equivalent to the assumption of a uniform temperature in the battery. If other geometries are used (thicker or cylindrical), the temperature inside the battery will become higher, and a one-dimensional simulation approach may not be valid anymore since a non-negligible part of the heat will flow to the other side surfaces of the battery. For applications with a large thermal strain on the battery, it is preferred to use battery with a limited thickness compared to the other dimensions. The heat flows in the battery are described by equation 4 . Heat generated by the battery can either be stored as sensible heat by an increase of the battery temperature or it can be transferred to the PCM structure $\left(\mathrm{q}_{\mathrm{c}}\right)$. The sensible heat is represented by the product of the battery mass $\mathrm{m}_{\mathrm{b}}$, the battery specific thermal capacity $c_{b}$ and the time derivative of the battery temperature.

$$
q_{b}=m_{b} c_{b} \frac{d T_{b}}{d t}+q_{c}
$$

3.2 PCM thermal model The thermal buffering of the battery heat is achieved through addition of PCMs. The simulation assumes a layer of PCM with uniform thickness in contact with both side surfaces of the battery. The PCM is assumed to be contained in a casing, one side in contact with the battery and the other side being cooled by natural or forced convection. A cross-sectional view of the battery, casing with PCM and the air gap where the cooling occurs is shown in Figure 5. The thermal behavior of the PCM is modelled as a one-dimensional thermal diffusion problem. Convection within the PCM is neglected, since the thickness of the PCM layers regarded here is small, resulting in conduction being the main heat transfer mechanism. One-dimensional flow of heat is described by equation 5 . The thermal diffusivity $\alpha$ is determined using equation 6 , where $\mathrm{k}$ is the thermal conductivity, $\rho$ is the density and $c_{p}$ is the specific heat capacity. To determine the temperature distribution in the PCM matrix, equation 5 is discretized in the length direction and in time.

$$
\begin{gathered}
\frac{\partial^{2} T}{\partial x^{2}}+\frac{1}{\alpha(T)} \frac{\partial T}{\partial t}=0 \\
\alpha(T)=\frac{k}{\rho c_{p}(T)}
\end{gathered}
$$




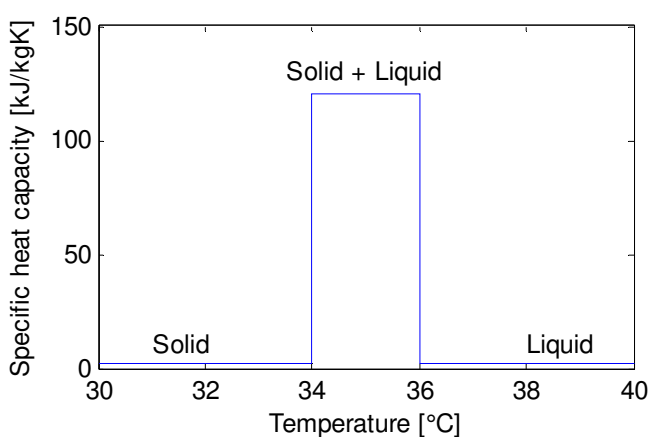

Fig. 6 Modelled specific heat capacity of 'RT35HC' in melting temperature range

The latent heat of melting and solidifying PCM is taken into account by making the specific heat capacity dependent on temperature. This is feasible since the PCMs used in these applications are mixtures which do not have a single melting point. The latent heat is released in a melting range. The profile used for the specific heat capacity is a rectangular profile, as in Figure 6. The properties of the PCM used in the simulations ('RT35HC' by Rubitherm) are summarized in Table 2. This is a paraffin based PCM with a melting range around $35^{\circ} \mathrm{C}$. To be able to compare simulations with different melting ranges without having to take into account differences in material properties, the properties of 'RT35HC' are also used in the following simulations with other melting ranges.

Table 2 Rubitherm 'RT35HC' PCM thermal characteristics

\begin{tabular}{lrl}
\hline Property & Value & Unit \\
\hline Melting range & $34-36$ & ${ }^{\circ} \mathrm{C}$ \\
Latent heat & 240 & $\mathrm{~kJ} / \mathrm{kg}$ \\
Solid/liquid specific heat capacity & 2000 & $\mathrm{~J} / \mathrm{kgK}$ \\
Solid density & 880 & $\mathrm{~kg} / \mathrm{m}^{3}$ \\
Liquid density & 770 & $\mathrm{~kg} / \mathrm{m}^{3}$ \\
Solid/liquid thermal conductivity & 0,2 & $\mathrm{~W} / \mathrm{mK}$ \\
\hline
\end{tabular}

The boundary condition of the differential equation at the battery side is the heat flow from equation 4 . The heat flow from the battery is halved since the problem is assumed to be symmetric, therefor halve of the heat will flow to one side of the battery. This is determined through the conductive resistances of the casing $\left(\mathrm{R}_{\text {cas }}=2,35 \mathrm{e}-4 \mathrm{~K} / \mathrm{W}\right)$ in which the PCM is contained and a thermal interface resistance $\left(R_{\text {int }}=0,0273 \mathrm{~K} / \mathrm{W}\right)$ between the battery and casing (equation 7). At the opposite side, the boundary condition is determined by conduction through the casing and natural or forced convection $\left(\mathrm{R}_{\text {conv }}\right)$ to the ambient air (equation 8). Natural convection on a vertical surface is modelled by a Nusselt-Rayleigh number correlation (equation 9), forced convection is modelled with the Gnielinski correlation [8], assuming air flows with a velocity of $5 \mathrm{~m} / \mathrm{s}$ in gaps between the battery cells of $5 \mathrm{~mm}$ width, which is representative for air cooled battery packs used in industry. Heating of the air flow is not taken into account in these simulations, since the temperature increase is limited compared to the temperature difference between battery and ambient (an increase of $3,5^{\circ} \mathrm{C}$ of air temperature from inlet to outlet in the most extreme case, where the temperature difference between battery and ambient is $30^{\circ} \mathrm{C}$ ). In these equations, $\mathrm{k}$ is the PCM thermal conductivity, A is the side surface of the battery, $\mathrm{T}(\mathrm{x})$ is the local PCM temperature, $\mathrm{T}_{\mathrm{amb}}$ is the ambient temperature $\left(30^{\circ} \mathrm{C}\right)$ and tPCM $_{\text {is }}$ the thickness of the PCM layer.

$$
\begin{array}{cc}
k A \frac{\partial T}{\partial x}=\frac{T_{b}-T(x)}{R_{\text {int }}+R_{\text {cas }}}=\frac{q_{c}}{2} & \text { at } x=0 \\
k A \frac{\partial T}{\partial x}=\frac{T(x)-T_{a m b}}{R_{c a s}+R_{\text {conv }}} & \text { at } x=t_{P C M} \\
N u=0.59 R a^{1 / 4} &
\end{array}
$$




\section{BATTERY THERMAL SIMULATION RESULTS}

4.1 Long cycle In the daily cycle, the maximal current is ten times lower than the maximum rated current of the battery. This results in low power losses of the battery and a small temperature difference between the battery and the ambient compared to cycles at nominal current. Due to the low cooling demand, natural convection is used for cooling. Figure 7 shows the variation of the battery temperature during the cycle. The battery heats up during discharging and cools down during charging. When there is no PCM applied, the maximum battery temperature is $35,4^{\circ} \mathrm{C}$ and the maximal temperature variation of battery is $9,9^{\circ} \mathrm{C}$. By adding a layer of PCM with a melting range from $30{ }^{\circ} \mathrm{C}$ to $32{ }^{\circ} \mathrm{C}$ and with a thickness of $1,5 \mathrm{~mm}$ on the sides of the battery, the maximum temperature can be reduced by $3,8^{\circ} \mathrm{C}$ and the temperature variation is reduced by $8,3{ }^{\circ} \mathrm{C}$. However, adding forced air cooling could also achieve a similar result.

4.2 Medium duration cycle In the medium duration cycle, the battery uses its full energy capacity and power density. This load cycle puts the most thermal strain on the battery. Natural and forced convection is compared when using no thermal buffering, the results are shown in Figure 8. When using natural convection cooling, the temperature of the battery can increase to nearly $80^{\circ} \mathrm{C}$, which can lead to thermal runaway. Therefor forced convection cooling is used in the following simulations. Figure 9 shows the temperature variation of the battery during the load cycle with PCM of melting range $35^{\circ} \mathrm{C}$ to $37^{\circ} \mathrm{C}$ and with different PCM layer thicknesses added. With no PCM added, the maximal temperature of the battery during the cycle is $58,8^{\circ} \mathrm{C}$. The amount of PCM used is scaled with the maximal amount of PCM that can be melted using the reversible heat of the battery. This amount relates to a PCM layer of 3,5 mm thick for this case (e.g. 100\% in Figure 9). If less PCM is used (e.g. 25\% in Figure 9), the PCM reaches temperatures out of the melting range, which results in higher battery temperatures. If more PCM is used (e.g. 200\% in Figure 9), part of the PCM will remain in solid or liquid state during the entire cycle, adding little extra thermal buffering but increasing the thermal resistance to the environment. The optimal amount of PCM is thus close to the amount that can be melted and solidified using the reversible heat of the battery. Table 3 shows the maximal battery temperature and the battery temperature variation, which is the difference between the maximal and minimal temperature during the entire cycle, as a function of the amount of PCM added. The highest reduction of the peak temperature is $12,9^{\circ} \mathrm{C}$, when there is less PCM than can be melted with the reversible heat. This reduces the thermal resistance while keeping adequate buffering. To reduce the temperature variation, more PCM is needed to increase the thermal mass.

Table 3 Peak battery temperature and temperature variation in function of PCM mass $\left(\mathrm{T}_{\mathrm{amb}}=30^{\circ} \mathrm{C}\right)$

\begin{tabular}{l|cccccccc}
\hline PCM mass (relative) & $0 \%$ & $25 \%$ & $50 \%$ & $75 \%$ & $100 \%$ & $125 \%$ & $150 \%$ & $200 \%$ \\
\hline Peak temperature $\left[{ }^{\circ} \mathrm{C}\right]$ & 58,8 & 55,3 & 49,0 & 45,9 & 46,1 & 46,3 & 47,1 & 49,8 \\
Temperature variation $\left[{ }^{\circ} \mathrm{C}\right]$ & 30,6 & 26,2 & 19,2 & 15,5 & 14,9 & 13,8 & 12,5 & 14,2 \\
\hline
\end{tabular}

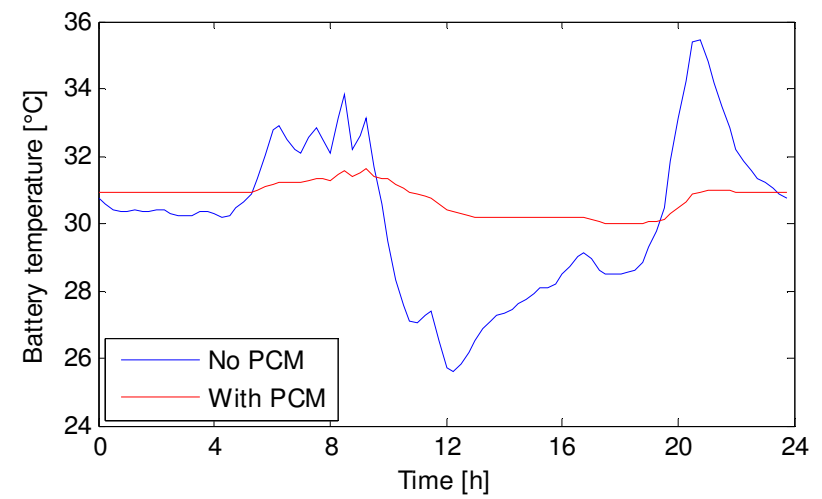

Fig. 7 Battery temperature during daily cycle with and without thermal buffering and natural convection cooling (ambient temperature of $30^{\circ} \mathrm{C}$ )

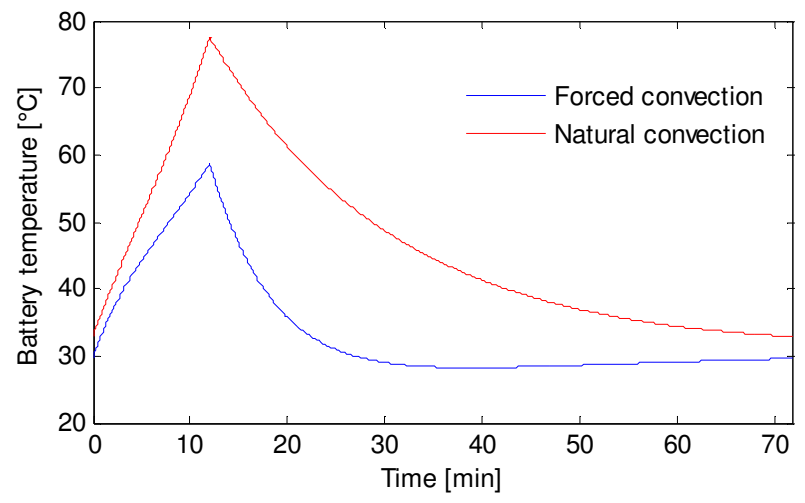

Fig. 8 Battery temperature 72 minute cycle without thermal buffering and natural and forced convection cooling (ambient temperature of $30^{\circ} \mathrm{C}$ ) 


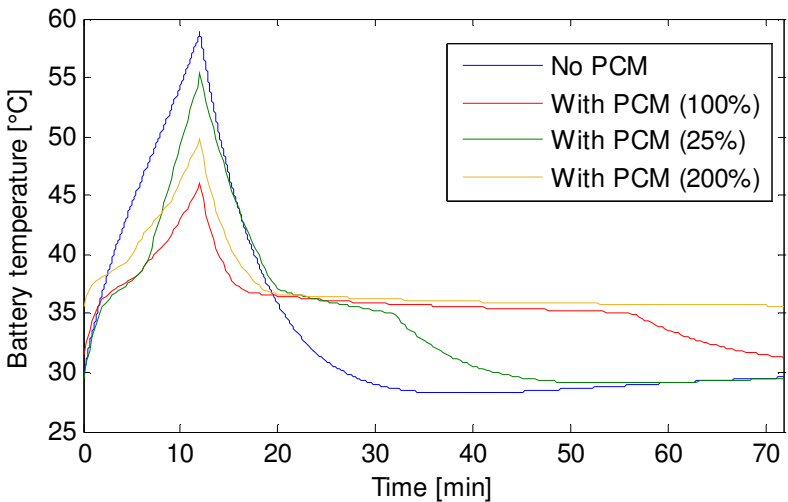

Fig. 9 Battery temperature during 72 minute cycle with and without thermal buffering with forced convection cooling (ambient temperature of $30^{\circ} \mathrm{C}$ )

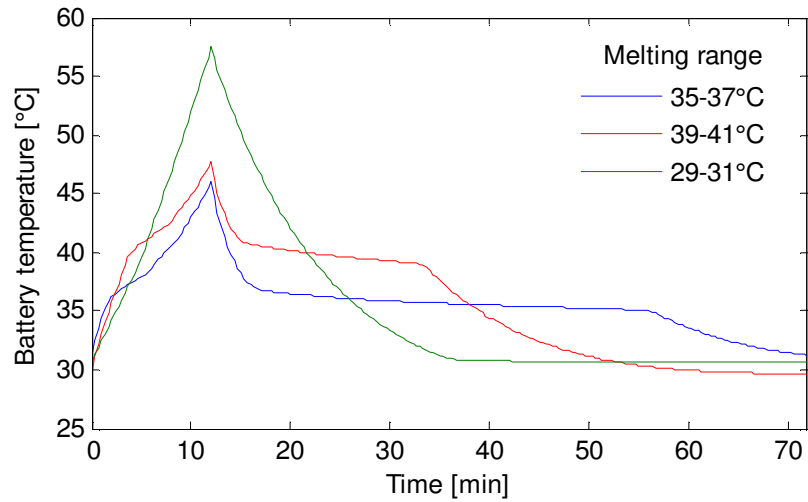

Fig. 10 Battery temperature during 72 minute cycle with different PCM melting temperatures and forced convection cooling (ambient temperature of $30^{\circ} \mathrm{C}$ )

The influence of the melting range on the battery temperature is shown in Figure 10. The optimal melting range is dependent on the load cycle and the heat transfer rate to the environment. With a melting range that is too low, part of the PCM will be liquid during the entire cycle and only act as an additional thermal resistance. If the melting point or range is too high, not all PCM will be melted during the cycle, but peak temperatures will still be buffered. It is therefore preferred to design the thermal buffer with a slightly higher melting point, since this design will still perform with higher loads, while only slightly decreasing the performance during nominal load.

Adding a conducting structure to PCM can enhance the thermal conductivity while preserving the thermal buffering effect. The battery temperature when utilizing a PCM structure combination is shown in Figure 11. The thermal conductivity of a metal foam structure is determined using the empirical correlation by Bhattacharya et al. [9]. In the simulations, an aluminum metal foam with a porosity of $95 \%$ is used, resulting in an effective thermal conductivity of $3 \mathrm{~W} / \mathrm{mK}$. For the PCM-expanded graphite matrix, thermal conductivity is based on the measurements by Sarı and Karaipekli [10], in the simulations a mass fraction of 10\% EG is added which results in an effective thermal conductivity of $0,82 \mathrm{~W} / \mathrm{mK}$. The thermal conductivity of PCM mixed with carbon fibers is based on measurements by Frusteri et al. [11], utilizing a 7\% carbon fiber mass fraction results in an effective thermal conductivity of $0,8 \mathrm{~W} / \mathrm{mK}$ which is used in the simulations. By adding expanded graphite or carbon fibers, the peak temperature can be reduced by $6,7^{\circ} \mathrm{C}$. For metal foam this reduction is $8,7^{\circ} \mathrm{C}$. However, the addition of a structure increases the complexity as well as the cost of the system. Furthermore, for these thin PCM layers (3,3 $\mathrm{mm}$ ) it might be infeasible or impossible to add these conducting structures.

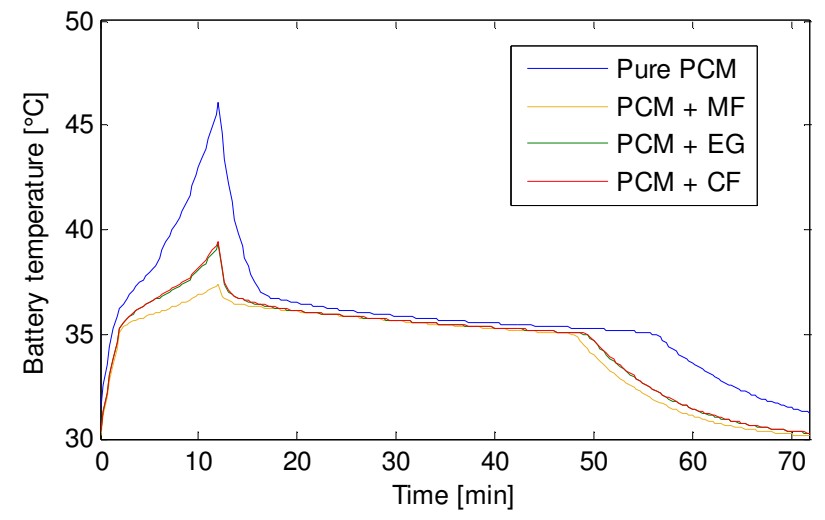

Fig. 11 Battery temperature during 72 minute cycle with PCM and conducting structures and forced convection cooling (ambient temperature of $30^{\circ} \mathrm{C}$ )

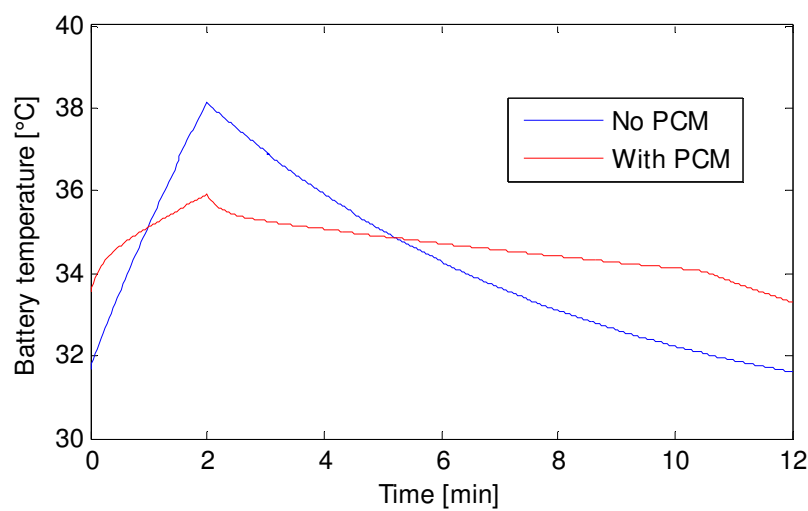

Fig. 12 Battery temperature during 12 minute cycle with and without thermal buffering with forced convective cooling (ambient temperature of $30^{\circ} \mathrm{C}$ ) 
4.3 Short cycle In the short load cycle, the battery is charged and discharged at the maximal rated current, but it only uses one sixth of its energy capacity. The average heat dissipation rate will be similar to those in the medium duration cycle, since the currents are equal, so forced convection cooling will be used. This results in an average temperature that is similar to that for the medium duration cycle. However, since the time period is shorter, the thermal mass of the battery will have a bigger effect and the temperature variation during the cycle will be smaller. Figure 12 shows the battery temperature during the load cycle with and without the usage of PCM. Using a PCM with melting range $34{ }^{\circ} \mathrm{C}$ to $36^{\circ} \mathrm{C}$ and a PCM layer thickness of $0,4 \mathrm{~mm}$, the maximal temperature can be reduced by $2,2^{\circ} \mathrm{C}$. The reversible heat in the cycle is already greatly buffered by the thermal mass of the battery itself. For these load cycles, the added cost and complexity of a thermal buffering system does not seem favorable.

\section{CONCLUSIONS}

A one-dimensional thermal model of a prismatic battery with thermal buffering utilizing phase change materials is made. The model is used to identify the influence of different boundary conditions and design parameters. By aiding the design of thermally buffered batteries, the efficiency of battery packs is increased and safety is improved. The results show that for daily load cycles, a design with natural convection and PCM thermal buffering can be useful. For cycles operating a the maximal current and energy capacity of the battery, a combination of forced convection and PCM can be used. The amount of PCM that can be melted using the reversible heat of the battery should be added for optimal buffering. Melting temperatures of the PCM are preferably chosen higher than optimal, to avoid overheating in extreme conditions. Thermally conductive structures can be added to the PCM to enhance the conductivity of the total system, however the benefit for adding these structures is small compared to the increased cost and complexity.

\section{ACKNOWLEDGMENT}

The results presented in this paper have been obtained within the frame of the TETRA project 150131 "Lithium-ion batteries for traction and stationary applications - LBATTS", funded by the Flemish Institute for Innovation and Entrepreneurship. This financial support is gratefully acknowledged.

\section{REFERENCES}

[1] Wang, Q., Ping, P., Zhao, X., Chu, G., Sun, J. and Chen, C., "Thermal runaway caused fire and explosion of lithium ion battery", J. of Power Sources, 208, pp. 210-224, (2012). Journal Paper

[2] Cabeza, L.F., Castell, A., Barreneche, C. D., De Gracia, A. and Fernández, A.I., "Materials used as PCM in thermal energy storage in buildings: a review", Renewable and Sustainable Energy Rev., 15(3), pp. 1675-1695, (2011). Journal Paper

[3] Al Hallaj, S. and Selman, J.R., "A Novel Thermal Management System for Electric Vehicle Batteries Using Phase-Change Material”, J. of the Electrochemical Society, 147(9), pp. 3231-3236, (2000). Journal Paper

[4] Khateeb, S.A., Amiruddin, S., Farid, M., Selman, J.R. and Al-Hallaj, S, "Thermal management of Li-ion battery with phase change material for electric scooters: experimental validation”, J. of Power Sources, 142(1), pp. 345-353, (2005). Journal Paper

[5] Kizilel, R., Sabbah, R., Selman, J. R. and Al-Hallaj, S., "An alternative cooling system to enhance the safety of Li-ion battery packs” J. of Power Sources, 194(2), pp. 1105-1112, (2009). Journal Paper

[6] Babapoor, A., Azizi, M. and Karimi, G., "Thermal management of a Li-ion battery using carbon fiber-PCM composites", Applied Thermal Engineering, 82, pp. 281-290, (2015). Journal Paper

[7] Nieto, N., Díaz, L., Gastelurrutia, J., Alava, I., Blanco, F., Ramos, J.C. and Rivas, A., "Thermal modeling of large format lithium-ion cells", J. of the Electrochemical Society, 160(2), pp. A212-A217, (2013). Journal Paper

[8] Gnielinski, V., "New equations for heat and mass transfer in turbulent pipe and channel flow", Int. Chem. Eng., 16(2), 359-368, (1976). Journal Paper

[9] Bhattacharya, A., Calmidi, V.V. and Mahajan, R.L., "Thermophysical properties of high porosity metal foams", Int. J. of Heat and Mass Transfer, 45(5), pp. 1017-1031, (2002). Journal Paper

[10] Sar1, A. and Karaipekli, A., "Thermal conductivity and latent heat thermal energy storage characteristics of paraffin/expanded graphite composite as phase change material", Applied Thermal Engineering, 27(8), pp. 1271-1277, (2007) Journal Paper

[11] Frusteri, F., Leonardi, V., Vasta, S. and Restuccia, G., "Thermal conductivity measurement of a PCM based storage system containing carbon fibers", Applied Thermal Engineering, 25(11), pp. 1623-1633, (2005). Journal Paper 